\title{
Modeling and validation of a boost pressure actuation system, for a series sequentially turbocharged SI engine
}

\author{
Andreas Thomasson, Oskar Leufvén, Ivan Criscuolo and Lars Eriksson
}

\section{Linköping University Post Print}

\section{Tweet}

N.B.: When citing this work, cite the original article.

Original Publication:

Andreas Thomasson, Oskar Leufvén, Ivan Criscuolo and Lars Eriksson, Modeling and validation of a boost pressure actuation system, for a series sequentially turbocharged SI engine, 2013, Control Engineering Practice, (21), 12, 1860-1870.

http://dx.doi.org/10.1016/j.conengprac.2013.01.004

Copyright: Elsevier / International Federation of Automatic Control http://www.ifac-control.org/

Postprint available at: Linköping University Electronic Press http://urn.kb.se/resolve?urn=urn:nbn:se:liu:diva-103723 


\title{
Modeling and validation of a boost pressure actuation system, for a series sequentially turbocharged SI engine
}

\author{
Andreas Thomasson ${ }^{\mathrm{a}, *}$, Oskar Leufvén ${ }^{\mathrm{a}}$, Ivan Criscuolo ${ }^{\mathrm{b}}$, Lars Eriksson ${ }^{\mathrm{a}}$ \\ a Vehicular Systems, Department of Electrical Engineering, Linköping University, SE-581 83 Linköping, Sweden \\ ${ }^{\mathrm{b}}$ Department of Mechanical Engineering, University of Salerno, Fisciano 84084, Italy
}

\section{A R T I C L E I N F O}

\section{Article history:}

Received 14 March 2012

Accepted 18 January 2013

Available online 22 February 2013

\section{Keywords:}

Engine

Turbocharger

Vacuum system

Solenoid valve

Nonlinear compensator

\begin{abstract}
A B S T R A C T
An actuation system for flexible control of an advanced turbocharging system is studied. It incorporates a vacuum pump and tank that are connected to pulse width modulation controlled vacuum valves. A methodology for modeling the entire boost pressure actuation system is developed. Emphasis is placed on developing component models that are easily identified from measured data, without the need for expensive measurements.The models have physical interpretations that enable handling of varying surrounding conditions.The component models and integrated system are evaluated on a two stage series sequential turbo system with three actuators having different characteristics.Several applications of the developed system model are presented, including a nonlinear compensator for voltage disturbance rejection where the performance of the compensator is demonstrated on an engine in a test cell. The applicability of the complete system model for control and diagnosis of the vacuum system is also discussed.
\end{abstract}

(c) 2013 Elsevier Ltd. All rights reserved.

\section{Introduction}

The trend toward downsizing of internal combustion engines in the automotive industry has increased in recent years. The main goal is to decrease fuel consumption and emissions, while keeping the performance of the engine constant. A way of achieving this goal is the introduction of turbocharging (Emmenthal, Hagermann, \& Hucho, 1979; Guzzella, Wenger, \& Martin, 2000; Petitjean, Bernardini, Middlemass, Shahed, \& Hurley, 2004). Lately, more than one turbo has been added to increase flexibility. Both series sequential (Chasse et al., 2008; Galindo, Climent, Guardiola, \& Domenech, 2009b; Zhang, Deng, Wang, \& Zhu, 2008) and parallel sequential Borila (1988) systems have been developed, and even three stage systems (Nitta, Minato, \& Shimazaki, 2011) have been evaluated. As turbocharging develops, the demand on the wastegate and bypass valve control strategies increases. Coordinated control of throttle, and compressor and turbine bypass valves is important, since the control affects engine performance and efficiency (Eriksson \& Nielsen, 2000; Eriksson, Frei, Onder, \& Guzzella, 2002). Boost control flexibility can be increased by including a vacuum tank and vacuum controlled actuators, that unlike pressure controlled actuators are unaffected by the current boost pressure. Both types are however affected by other

\footnotetext{
* Corresponding author. Tel.: +4613286862.

E-mail addresses: andreast@isy.liu.se (A. Thomasson), oleufven@isy.liu.se (O. Leufvén), icriscuolo@unisa.it (I. Criscuolo), larer@isy.liu.se (L. Eriksson).
}

surrounding conditions, like system voltage and ambient pressure. This leads to an increasing need for good knowledge about the complete vacuum system and how surrounding conditions affect boost control performance. Unnecessary operation of the high power vacuum pump, naturally also affects fuel efficiency, due to its increased electrical load.

\subsection{Related research and contributions}

A control strategy for a pneumatic system, based on using actuator acceleration feedback is presented in Wang, $\mathrm{Pu}$, and Moore (1999). No air leakages, constant supply pressure and measurement of actuator position to estimate acceleration was assumed. A self-tuning LQG pressure regulator for a pneumatic pressure load systems is presented in Wang, Cheng, and Peng (2007). Compared to the control strategy in Wang et al. (1999), the requirement of acceleration feedback is removed. The valve flow is modeled with valve position dependent effective flow areas, and the actuator is modeled using an emptying and filling approach. Constant supply pressure and no leakage are assumed in these papers, while this paper contributes by considering varying pressure and models the leakage flow.

To increase controller response, a Pulse Width Modulation (PWM) peak and hold control strategy is proposed in Amirante, Innone, and Catalano (2008). The approach presented removes the requirement for a control valve position sensor, but instead requires a special power driver, and is shown to increase the performance of the control valve. 
A switching valve is developed and modeled in Topcu, Yüksel, and Kamis (2006), describing the underlying electromagnetic subsystems of the control valve. The very rapid valve switching times, and thus PWM frequency, is emphasized, as well as the good linearity between PWM frequency and valve position. Physical modeling of the electromagnetic solenoid system is further presented. The pneumatic actuator system of an automotive VGT system is modeled and simulated in Mehmood, Laghrouche, and El Bagdouri (2011), emphasizing and modeling the aerodynamic forces due to the exhaust gas and friction hysteresis effects. The model assumes no leakages, isothermal processes and then describes the mass flow between system components. The modeling makes use of force sensors. The aerodynamic forces are also discussed in Naseradinmousavi and Nataraj (2011), along with physical modeling of the electromagnetic properties.

These referenced papers contribute with in depth knowledge of the physical properties of the valves and pneumatic actuator. This paper builds upon these results, and develops a novel model connecting the subsystems while reducing the need for detailed solenoid measurements, geometric data, and magnetic properties In particular it extends the results of Criscuolo, Leufven, Thomasson, and Eriksson (2011), and the main contribution is a physically based system model for the pneumatic actuator system that is easy to tune from measurements. The model balances model complexity and capability to describe the system, from a boost pressure control perspective. Compared to Criscuolo et al. (2011) the analysis is extended, and a generalized modeling methodology is developed. The methodology is shown to be applicable to all three actuators of a series sequential two stage charging system, and thus also includes the compressor bypass valve. Further, the paper contributes with knowledge about the spread among components. The modeling covers the full boost pressure actuator system, and therefore also includes models for the vacuum pump and tank subsystems. The vacuum pump is especially important since it consumes high power, and therefore affects the system supply voltage and thereby the boost pressure control. The model structure and parameterization methods are applicable for complex boost pressure control development.

The proposed system model can be integrated in an MVEM structure. MVEM was coined in Hendricks (1989), and component based MVEM of Turbo Charged (TC) Spark Ignited (SI) engines is outlined in Eriksson, Nielsen et al. (2002). The baseline model is developed and validated in Andersson (2005) and Eriksson (2007).

\subsection{Outline}

The engine and system components are described in Section 2, together with the experimental setup. The data collected for modeling, together with the development of a model for the actuator and solenoid valves, including parameter identification and model validation, are described in Section 3. Section 4 describes the vacuum tank and vacuum pump model, including validation of the models for the different mass flows to and from the tank. In Section 5 several application examples of the developed system model are presented. A nonlinear compensator for voltage disturbance rejection is presented and validated on an engine in a test cell. The possibility to use the model for diagnosis of the vacuum system is discussed, and the contribution to a model based boost control system is pointed out. Conclusions are presented in Section 6. A nomenclature is included in Appendix A.

\section{Experimental setup}

This section describes the series sequential two stage turbocharged engine, its actuation system and the experimental setup.

\subsection{Engine and control system}

The measurements have been performed in the engine laboratory at the division of Vehicular Systems, Linköping University. A 2.01 GM four cylinder direct injection spark ignition engine, equipped with an experimental series sequential two stage system was used for the experiments. The advantage of this layout comes from the different sizes of the turbochargers, where the turbo of the low pressure (LP) stage is larger than the one of the high pressure (HP) stage. In this way, it is possible to utilize each turbocharger in its region of maximum efficiency.

The engine uses a rapid prototyping system from dSpace (RapidPro and MicroAutoBox), connected to a PC running ControlDesk. An overview of the actual two stage system installation, and the vacuum tank with its pump and the three solenoid valves used for control, is shown in Fig. 1. System voltage disturbances are injected into the system using an array of light bulbs, controlled by the ECU. A schematic overview of the components and signals are given in Fig. 2.

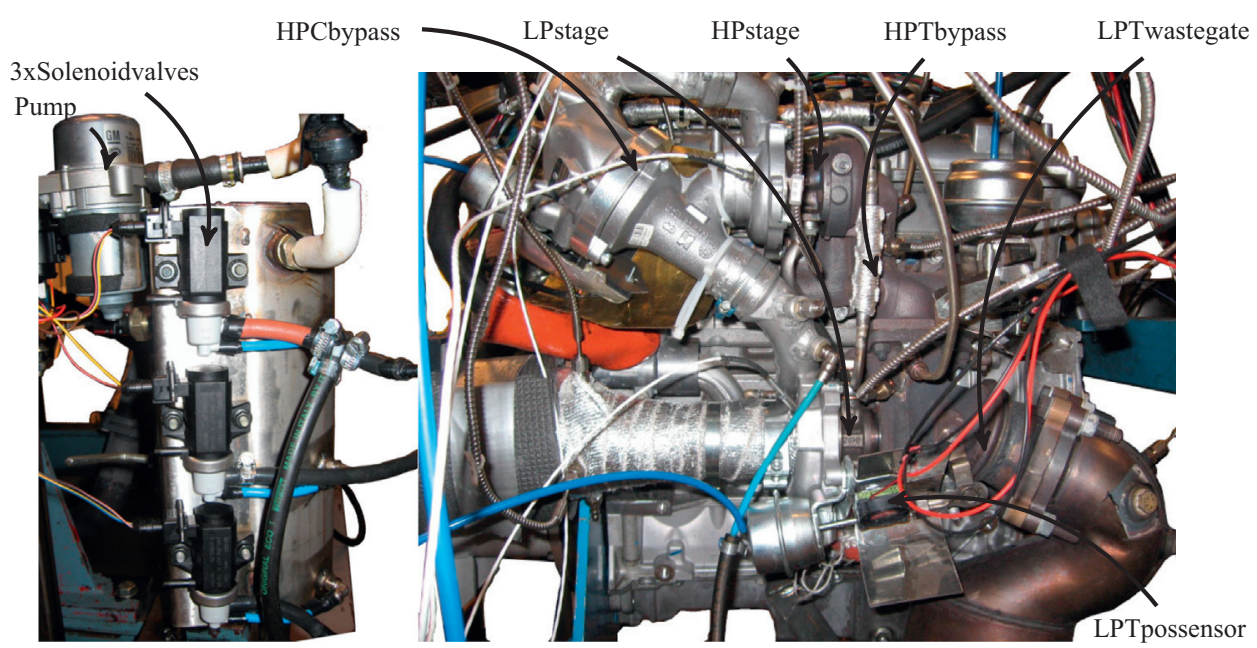

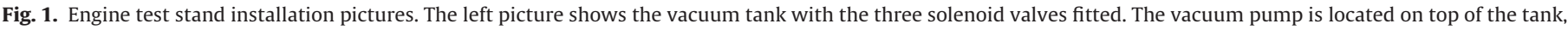

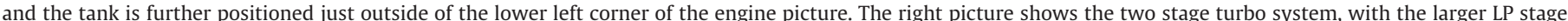
and the smaller HP stage, as well as the position sensors. 


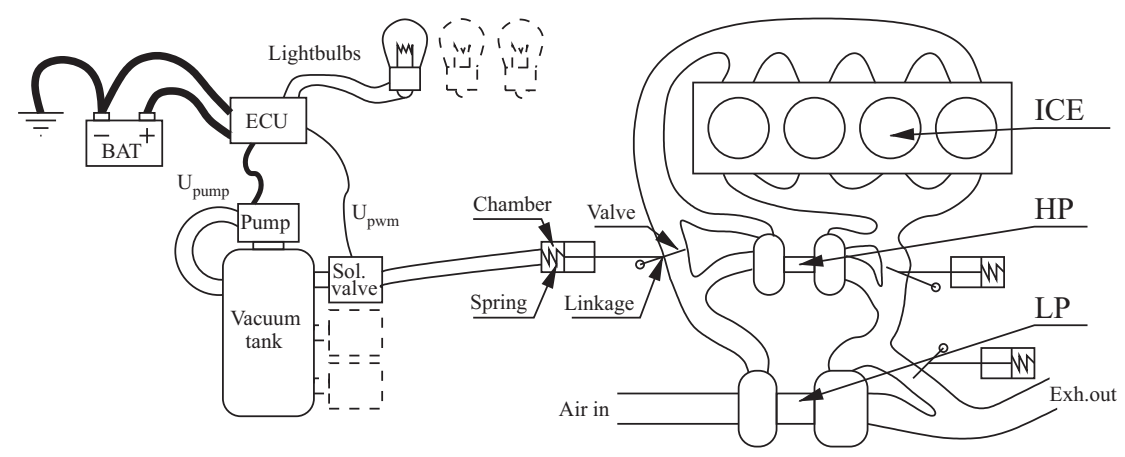

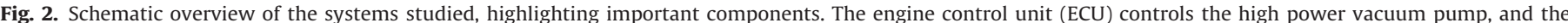

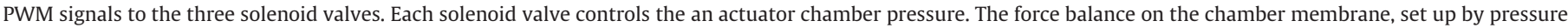

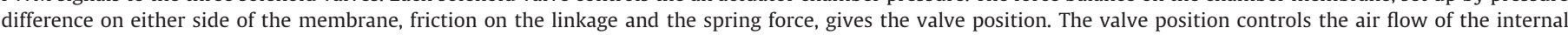

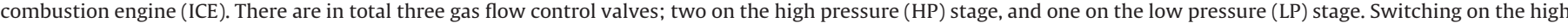
power pump affects the supplied voltage to the ECU from the battery (BAT).
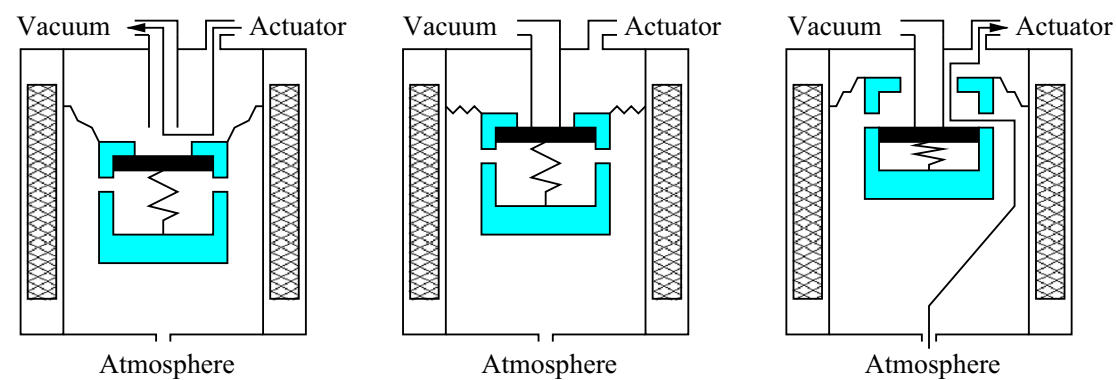

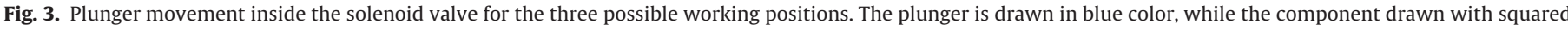

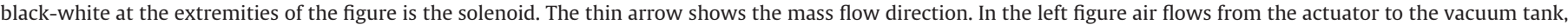

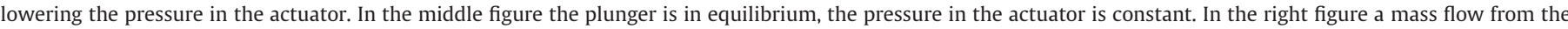

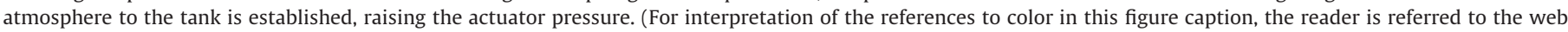
version of this article.)

\subsection{Boost pressure actuation system}

The actuation system consists of three different pneumatic actuators, each supplied by a PWM controlled solenoid valve. In this work, the frequency of the PWM signal was $300 \mathrm{~Hz}$, and the PWM duty cycle is updated by the controller at $80 \mathrm{~Hz}$. The high pressure stage can be by-passed both on the compressor and the turbine side, and a waste gate valve is used for the low pressure stage turbine. All three actuators consist of a membrane sealed chamber, where a controllable vacuum pressure acts on one side of the membrane, and ambient pressure on the other. The pressure difference creates a force. A spring is further used to control the membrane to a safe default position. The membrane is connected via a rod, to the actual valve controlling the engine flow. The geometries of both the rod linkage and the membrane and actuator chamber differ between the three actuators. The solenoid valve contains a plunger, which position is controlled by a PWM signal. The plunger position then controls the pressure in the other pneumatic components. All three actuators use the same solenoid valve type.

The system further consists of a vacuum tank with a high power vacuum pump. The pressure in the vacuum tank should be kept low enough, to allow the membrane force to overcome the other actuator forces, and thereby allowing fully opened and closed valves. The vacuum tank pressure is controlled by the control system, which switches on the vacuum pump when the pressure becomes too high. The reasons for tank pressure rises are leakages and plunger movement in the valve. Leakages are due to air infiltration through the ducts of the system and the elastic membrane in the solenoid valve. Further, when the plunger is in the down position, a mass flow from actuator to tank is established increasing the tank pressure, seeFig. 3. More information on plunger behavior can be found in Galindo, Climent, Guardiola, and Domenech (2009a) and Mehmood, Laghrouche, and El Bagdouri (2010).

The dSpace equipment was used to both measure the relevant signals (position and pressure) and generate the PWM valve control signals, and to control the system supply voltage using external circuits. Blade 25 position sensors from Gill instruments were used for the two waste gate valves on the turbine side, and a model 9610 linear position sensor from BEI sensors was used for the HP stage compressor bypass. The sensors used to measured actuator pressures were Kistler models 4260 and 4295. The tank pressure was measured using the built in production sensor, which was calibrated using the Kistler cell sensors.

\section{Actuator modeling}

This section presents a modeling methodology for vacuum controlled actuators, commonly used to control turbochargers in the automotive industry. The characteristics of these actuators can vary significantly but their common physical structure allows the same method and model structure to be applied.

The models structure is divided into three parts, the pressure solenoid model, i.e. the relationship between control signal and actuator pressure, a model for the actuator pressure to position and a model for the mass flow leakage to the vacuum tank. The last part is important for a complete system understanding, since the vacuum pump consumes high power, affecting the supply voltage and thereby the solenoid valve characteristic. The models require neither detailed knowledge of electrical and magnetic 
properties of the solenoid nor the plunger movement inside it, but are easily identified from measurements and applicable to control design. The only extra sensors used are actuator chamber pressure and position of the connecting rod.

To demonstrate the methodology, each submodel is identified for all three vacuum actuators on a series sequential turbocharging system. In each subsection the model are presented together with identification for one wastegate actuator, followed by the corresponding plots for the other wastegate and bypass actuator, and discussion of similarities and differences. The nomenclature used in the following sections is found in Appendix A.

\subsection{Control signal to pressure}

The actuator pressure is governed by a solenoid valve connected to the vacuum tank, ambient pressure, and the actuator, see Fig. 3. The solenoid is controlled by the ECU with a PWM signal. Depending on the duty cycle the solenoid will strive for a certain pressure drop from the atmosphere to the actuator. This is done by either leading air form the actuator to the vacuum tank, lowering the pressure in the actuator, or by leading air from the atmosphere to the actuator, raising the actuator pressure.

A slow ramp response up and down in control signal is shown to the left in Fig. 4. It is evident that different system voltages give different characteristics of the control signal to pressure relation. Normalizing the control signal with the system voltage results in a control signal with the same average value for different supply voltage. The corrected control signal, $u_{\text {corr }}$, becomes

$u_{\text {corr }}=\frac{U_{\text {syst }}}{U_{\text {ref }}} u$

where $u$ is the control signal, $U_{\text {syst }}$ is the system voltage and $U_{\text {ref }}$ is a reference voltage. The right figure shows the actuator pressure plotted against corrected control signal, $u_{\text {corr }}$. The difference between the curves depends on whether the pressure is increasing or decreasing. The mean value of the two curves is identified as the static control signal to pressure relation. For this actuator the relationship is almost linear in control signal, with a change in proportionality constant around $u_{\text {corr }}=25$. It is therefore described as a piecewise linear function in control signal, saturated above and below by the ambient pressure, $p_{a m b}$, and the vacuum tank pressure, $p_{v a c}$, respectively.

$$
\begin{gathered}
p_{L P T}^{*}=\min \left(k_{1} u_{\text {corr }}+p_{01}, k_{2} u_{\text {corr }}+p_{02}\right) \\
p_{L P T, s t a t}= \begin{cases}p_{a m b} & \text { if } p_{L P T}^{*} \geq p_{a m b} \\
p_{L P T}^{*} & \text { if } p_{v a c} \leq p_{L P T}^{*} \leq p_{a m b} \\
p_{v a c} & \text { if } p_{L P T}^{*} \leq p_{v a c}\end{cases}
\end{gathered}
$$

Model parameters are the proportionality constants, $k_{1}$ and $k_{2}$, and the offsets $p_{01}$ and $p_{02}$, for the linear functions. The model output is the static pressure, $p_{L P T, \text { stat }}$. In the right plot of Fig. 4 this model has been fitted to the corrected control signal to pressure data, and it can be seen that the agreement is good.

The actuator pressure does not change instantaneously as the control signal changes. The pressure rise depends on the mass flow between ambient, actuator and vacuum tank, and can be modeled as

$\dot{m}=\frac{p_{u}}{\sqrt{R T_{u}}} C_{d} A \Psi\left(\Pi_{\text {lim }}\right)$

$\Psi\left(\Pi_{\text {lim }}\right)=\sqrt{\frac{2 \gamma}{\gamma-1}\left(\Pi_{\text {lim }}^{2 / \gamma}-\Pi_{\text {lim }}^{(\gamma+1) / \gamma}\right)}$

$\Pi_{\text {lim }}=\max \left(\Pi,\left(\frac{2}{\gamma+1}\right)^{\gamma /(\gamma-1)}\right)$

where $\dot{m}$ is the mass flow, $p_{u}$ and $T_{u}$ are the pressure and temperature upstream, $R$ is the specific gas constant, $C_{d}$ is the discharge coefficient, $A$ is the flow area, $p_{d}$ is the pressure downstream and $\Pi=p_{d} / p_{u}$ is the pressure ratio, see e.g. Taghizadeh, Ghaffari, and Najafi (2009). This model requires detailed knowledge about the solenoid and how the effective area $A_{\text {eff }}=C_{d} A$ varies with control signal and pressures.

Analyzing the operating principles and interaction between the solenoid valve and the actuator pressure, presented in e.g. Topcu et al. (2006), Mehmood et al. (2010) and Galindo et al. (2009a), an opportunity for model reduction is identified. It is recognized that the solenoid valve acts as a controller for the actuator pressure, where the reference in this controller is $p_{L P T, \text { stat }}$. This is motivated by the property that the solenoid controls the pressure toward the static pressure in Eq. (2) and Fig. 4. Studying measurements, it is seen that a first order system is a good approximation for the pressure dynamics, see Fig. 5. A simple model for this is therefore

$\tau \dot{p}_{L P T, d y n}=-p_{L P T, d y n}+p_{L P T, s t a t}$

where $p_{L P T, s t a t}$ is the input pressure, $p_{L P T, d y n}$ is the output pressure and $\tau$ is the time constant for the first order dynamic system. This model is easily identified from measured data with few extra sensors, using ramps and step responses in control signal.

Fig. 5 shows two step responses in control signal, together with a step response for a first order system. The pressure response to a step is well described by the first order model, but different time constants have to be used for increasing and decreasing pressure. This difference is due to different effective areas and pressures for the mass flow from ambient to actuator, compared to actuator to vacuum tank.
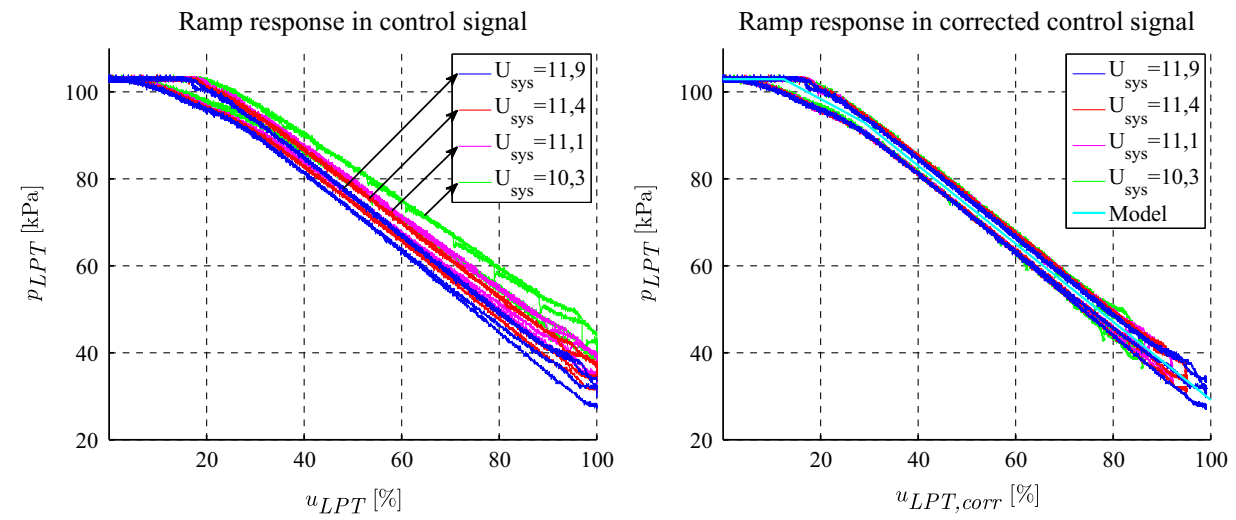

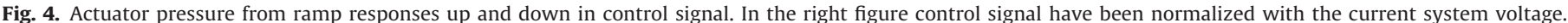
When this is done the control signal to pressure relation align into two curves, determined by whether the pressure is increasing or decreasing. 

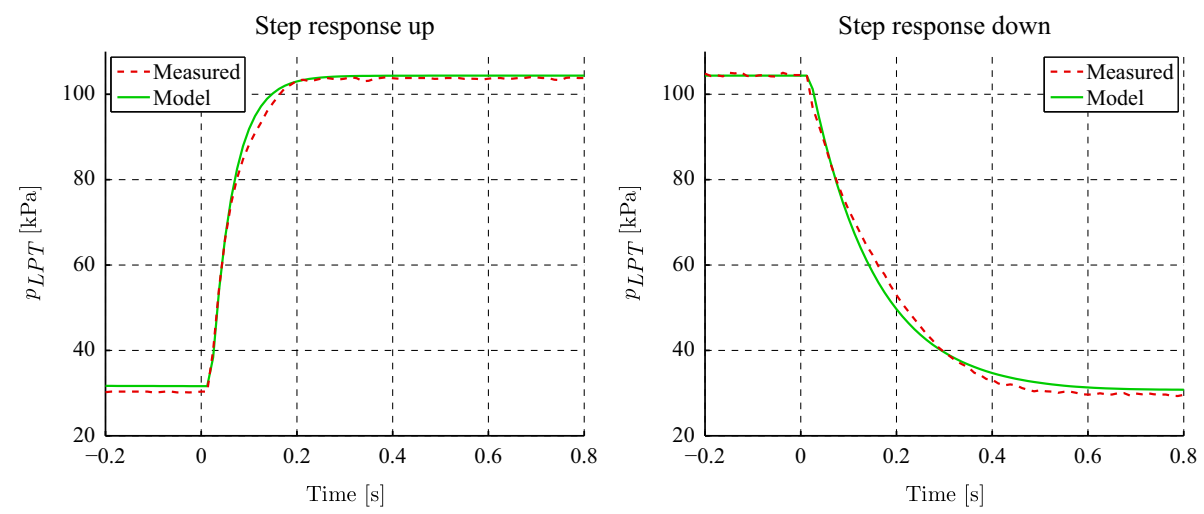

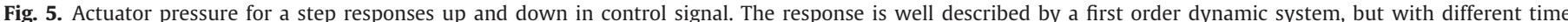
constants for increasing and decreasing pressure.
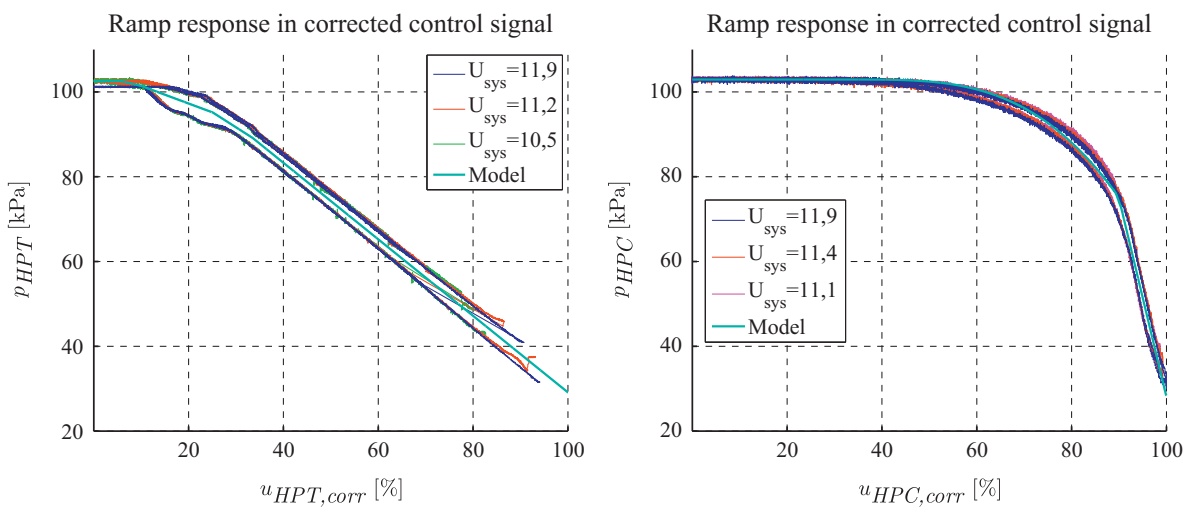

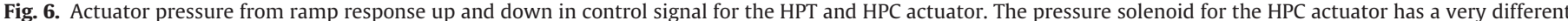
characteristic than the other two actuators. The model structure remains the same, but a different function for the static pressure is used.
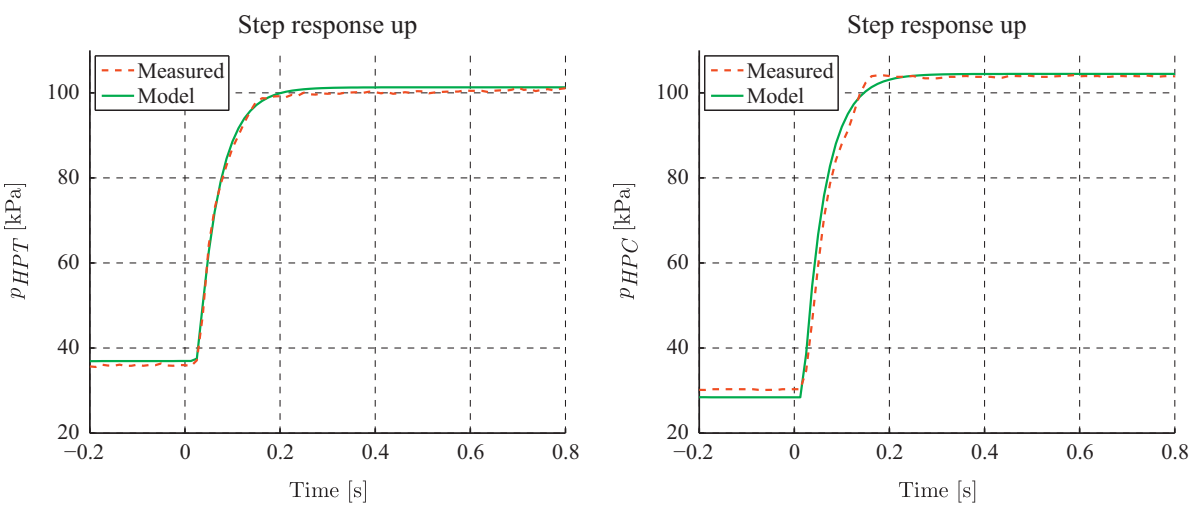

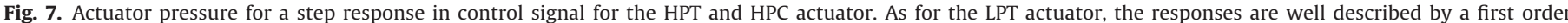
dynamic system.

Ramp responses for the other two actuators are shown in Fig. 6, where measured and modeled pressure is plotted against corrected control signal. The characteristics of the HPC solenoid is very different, compared to that of the other two. This requires another function to be used for the static pressure, but the same model structure can be used.

$p_{H P C}^{*}=\min \left(k_{1} u_{\text {corr }}+p_{01}, p_{a m b}+k_{2} \max \left(0, u_{\text {corr }}-u_{0}\right)^{3}\right)$

$$
p_{H P C, \text { stat }}= \begin{cases}p_{a m b} & \text { if } p_{H P C}^{*} \geq p_{a m b} \\ p_{H P C}^{*} & \text { if } p_{v a c} \leq p_{H P C}^{*} \leq p_{a m b} \\ p_{v a c} & \text { if } p_{H P C}^{*} \leq p_{v a c}\end{cases}
$$

The parameters $u_{0}$ and $k_{2}$ describe the decrease from ambient pressure at $u_{\text {corr }}=u_{0} \approx 40 \%$ until $u_{\text {corr }} \approx 90 \%$, and $k_{1}$ and $p_{01}$ describes the linear region with $u_{\text {corr }} \approx 90-100 \%$, see the right plot of Fig. 6.

The pressure dynamics for the other two actuators are shown in Fig. 7. As for the LPT actuator solenoid the dynamics is well captured by a first order dynamical system.

\subsection{Pressure to position}

The pressure in the actuator chamber exercises a force on the membrane in the actuator. The other forces are the spring force, the friction force and forces resulting from the gas in the exhaust or intake acting on the wastegate or bypass valve respectively, see Fig. 8. This means that the model does not only depend on the actuator itself, but is also affected by the mounting on the engine. 
The sum of the forces on the actuator accelerates the connecting rod of the actuator and the valve it is connected to, according to Newton's second law of motion

$m \ddot{x}=F_{a m b}-b \dot{x}-F_{a c t}-F_{f r}-F_{s p}-F_{a e r o}$

where $x$ is the displacement, $m$ is the mass of the moving parts, $b$ is the dynamic friction coefficient, $F_{a c t}$ is the force from the actuator pressure on the membrane, $F_{a m b}$ is from the ambient pressure on the other side of the membrane, $F_{s p}$ is the spring force, and $F_{\text {aero }}$ is the aerodynamic force. To accurately identify the relation between the forces $F_{a m b}, F_{a c t}, F_{s p}, F_{f r}$ it is recommended to run the experiments without the engine running. To model $F_{\text {aero, }}$ one can e.g. follow the approach presented in Mehmood et al. (2010).

A slow ramp response in pressure can be used to identify the spring and static friction forces. Fig. 9 shows position plotted against pressure for ramp responses in control signal for the LPT actuator, previously used to identify the static pressure relation. The ramp responses are very slow and approximates the static relation given by setting $\ddot{x}=0$ and $\dot{x}=0$ in (6). To further simplify identification, since $F_{a m b}=p_{a m b} A_{a c t}$ and $F_{a c t}=p_{a c t} A_{a c t}$ where $A_{a c t}$ is the membrane area, the force balance is normalized with $A_{\text {act }}$ to

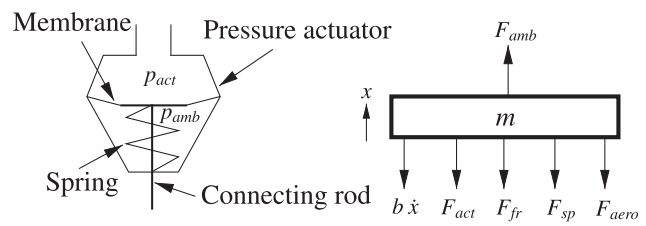

Fig. 8. Actuator working principle and the forces acting on the mechanical system.

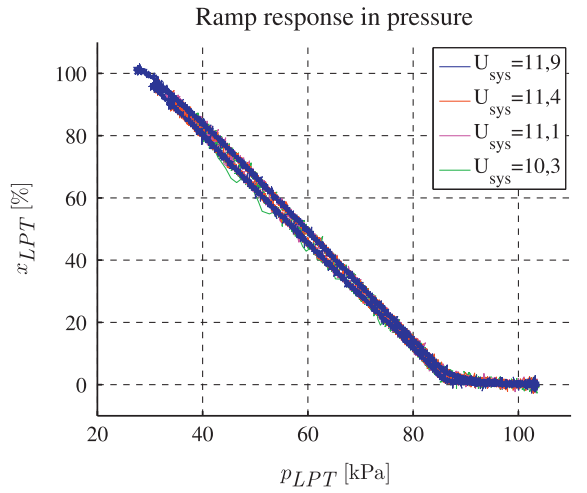

get the relation

$0=p_{a m b}-p_{a c t}-p_{s p}\left(x_{a c t}\right)-p_{f r}$

Assuming that the friction has the same magnitude in both directions, $p_{f r}$ can be estimated as the difference between the ramps up and down, and the mean of the two ramps with $p_{f r}=0$ gives $p_{s p}\left(x_{a c t}\right)$. In the right plot of Fig. 9 the following linear model for the LPT actuator position is included

$x_{L P T}=k p_{L P T}+x_{0}$

where $k$ and $x_{0}$ are model parameters. From (7) and (8), $p_{s p}\left(x_{a c t}\right)$ is easily determined ( $x_{a c t}=x_{L P T}$ for this actuator).

Ramp responses for the other two actuators are shown in Fig. 10. Two differences compared to the LPT actuator are evident. The relationship is not linear and the distance between the ramp up and down, corresponding to the friction force, is greater and varies with position.

Measurements from ramps up and down are used to parameterize two static relations from pressure to position, see Fig. 10. The mean value is then used as the spring force (or pressure) and half the distance between them as a position dependent friction. To parameterize the pressure position relation for the two curves, for both actuators an arctan function has been used.

$x_{a c t}=a_{1}+a_{2} \arctan \frac{a_{3}-p_{a c t}}{a_{4}}$

The parameters $a_{1}$ and $a_{3}$ determine the xy-position of the curve and $a_{2}$ and $a_{4}$ controls the shape. All parameters are estimated from measurements using least squares. In the same manner as for the LPT actuator, given a position this is used to calculate the resulting spring force or pressure. The difference being that two

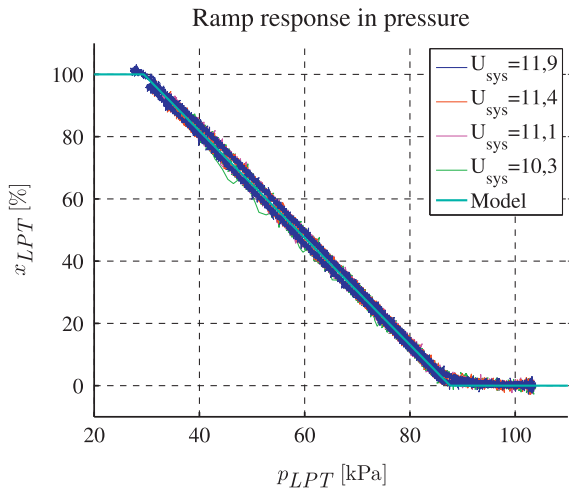

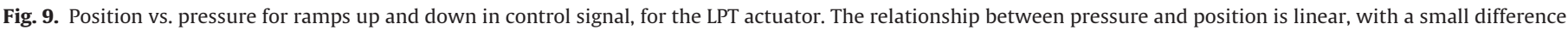
between ramps up and down due to friction. In the right figure a linear model with saturation is included.
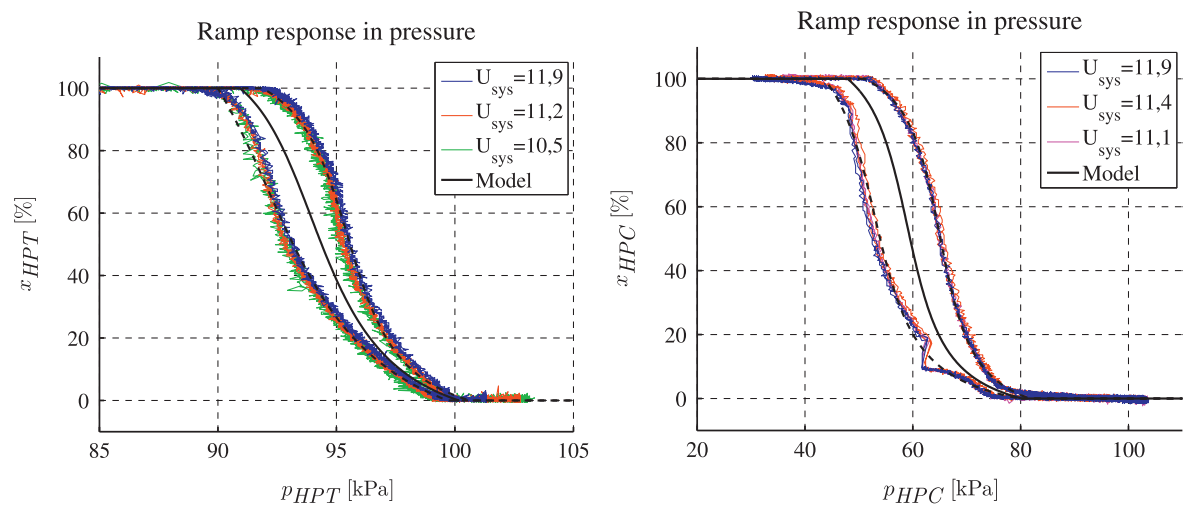

Fig. 10. Position vs. pressure for ramps up and down in control signal for the HPT and HPC actuators. For both these actuators, a larger friction is present. 

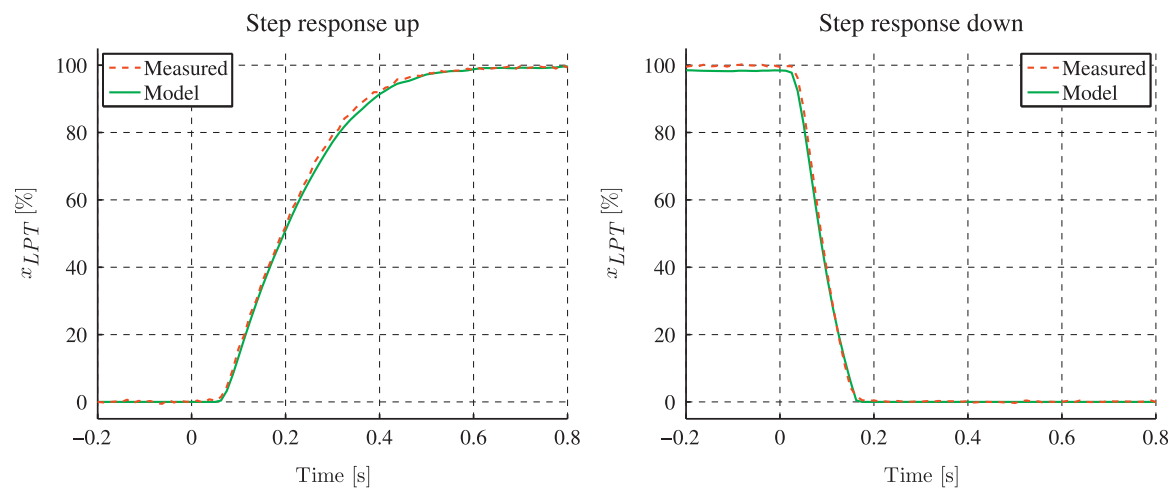

Fig. 11. Measured and modeled pressure response for a step up and down in control signal for the LPT actuator.
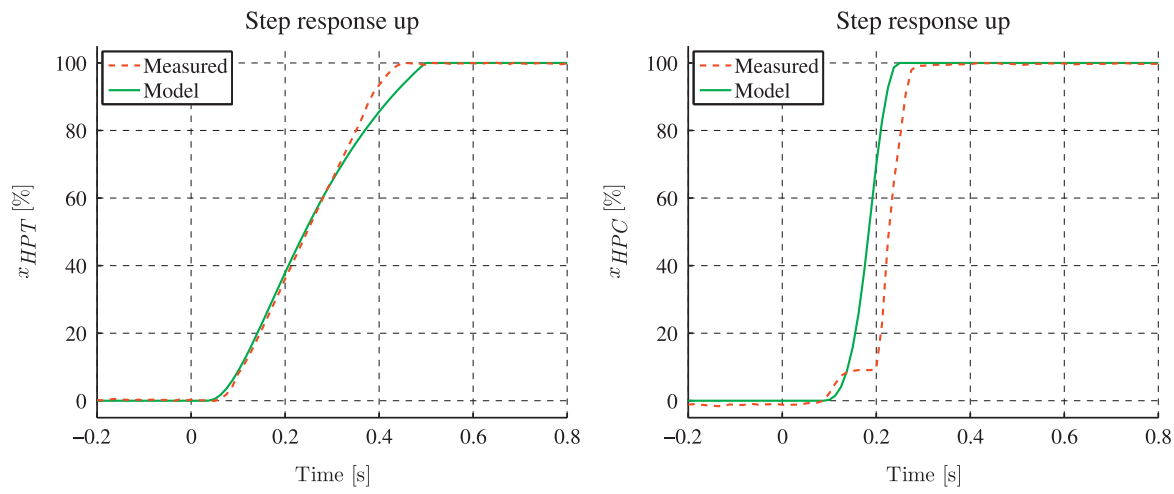

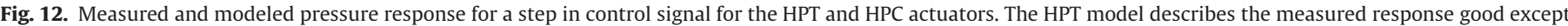

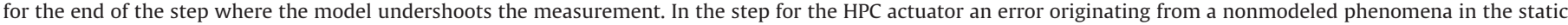

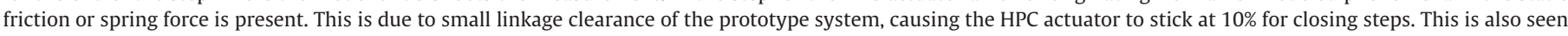
in Fig. 10.

pressures are calculated and the mean is used for the spring force and half the difference as the coulomb friction.

To model the friction force the popular Dahl's model is used, proposed by Dahl (1968) and used also by e.g. Singh and Kunt (1990), Hlouvry and Dupont (1994), and Mehmood et al. (2010):

$\frac{d F_{f r}}{d t}=\sigma\left(1-\frac{F_{f r}}{F_{c}} \operatorname{sgn}(\dot{x})\right)^{\alpha} \dot{x}$

where $F_{c}, \sigma$ and $\alpha$ are model parameters that determine the shape of the curve. Further, the common choice of $\alpha=1$ is used, see Olsson, Åström, Canudas de Wit, Gäfvert, and Lischinsky (1998).

The position dynamics are determined by the parameters $m$ and $b$ in (6). These are identified using the least squares method from measured pressure and position for steps in control signal. The dynamic friction for the step responses up and down is different, roughly by a factor of two. Measured and modeled LPT actuator positions, for steps up and down in control signal, are shown in Fig. 11. It is seen that the mass spring damper model gives a good description of the measured data.

The same procedure can applied to identify the parameters of the dynamic position response for the other two actuators. Step responses for these actuators are shown in Fig. 12. The step response for the HPT actuator follows the measured position, except at the end of the step where the modeled position is lower. In the step for the HPC actuator a nonmodeled phenomena is present, the actuator almost comes to a halt soon after the step starts. This is due to small linkage clearance of the prototype system, causing the HPC actuator to stick at $10 \%$ for closing steps. This results in an error in the static friction force and is also evident in the ramp response in the right plot in Fig. 10.

\subsection{Mass flow}

To model the complete vacuum system, the mass flow leakage from the actuators and surrounding to the vacuum tank needs to be estimated. This is divided into different parts. First a base leakage is determined by only looking at the pressure rise in the vacuum tank without operating the solenoid valves. The leakage flow is modeled with the orifice equation for compressible flow (3), and an effective leakage area is determined.

Ideally there should be no more leakage than this for constant control signal when the actuator pressure has stabilized, and the plunger in the solenoid has reached equilibrium. The plunger will however oscillate and allow some flow through the solenoid valve. The left plot of Fig. 13 shows that the leakage flow for constant control signal increases with increasing control signal. The right plot of Fig. 13 shows the effective area for the leakage flow from actuator to vacuum tank plotted against control signal. The area increases linearly until approximately $80 \%$ control signal, a linear model is also included in the figure. In the next section it is shown that this model is sufficient to give an accurate enough estimate of the leakage flow for constant control signal. If more accuracy is needed, a more complicated function or look-up table can be used.

The last part of the mass flow from actuator to tank is due to changes in pressure and volume of the actuator. To reduce the pressure in the actuator a mass flow is established from the actuator to the tank. In addition when the pressure decreases the actuator volume is reduced which also contributes to the mass flow to the tank. Starting from the ideal gas law

$p_{a c t} V_{a c t}=m_{a c t} R T_{a c t}$ 

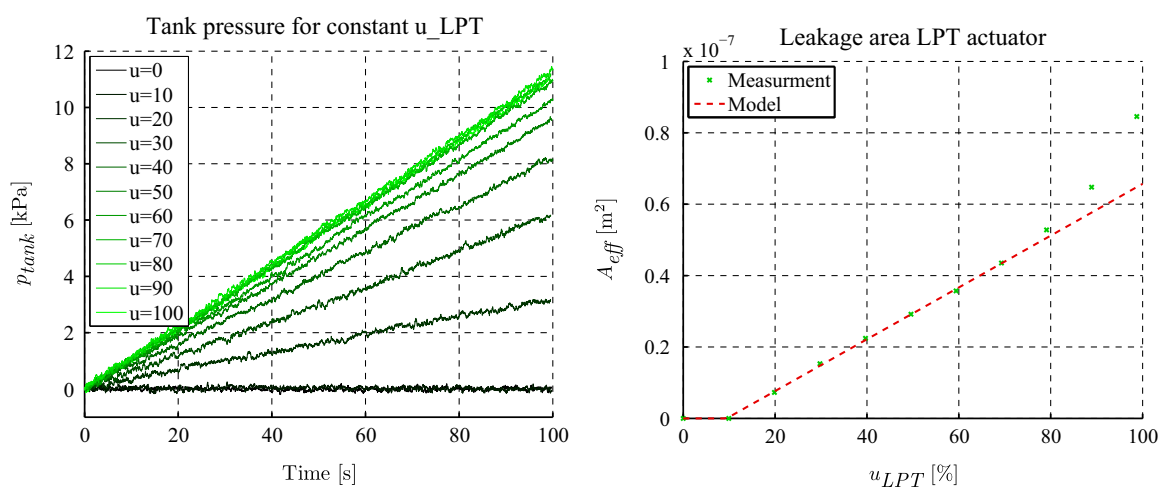

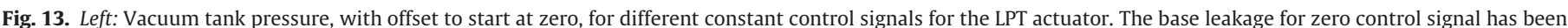

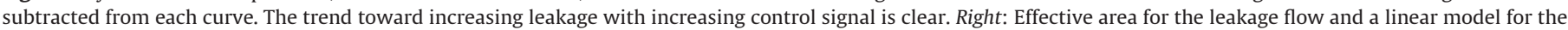
effective area. The model is very accurate up to $80 \%$ control signal.

constant temperature is assumed, $T_{a c t}=T_{a m b}$. Differentiating and saturating the result at zero from below, since negative mass flow will mean mass flow from ambient to actuator, gives the following equation for the mass flow from actuator to tank

$\frac{d m}{d t}=\max \left(0, \frac{1}{R T_{a m b}}\left(\frac{d p_{a c t}}{d t} V_{a c t}+\frac{d V_{a c t}}{d t} p_{a c t}\right)\right)$

The procedure for identifying the mass flow leakage from the other two actuators are identical and is not included here. Validation of the mass flow leakage models for all actuators is done together with the vacuum tank model in the next section.

\section{Vacuum tank and pump model}

The vacuum tank provides a low pressure reservoir needed to operate the vacuum actuators. This part is important for the complete system, since it determines how often the vacuum pump will be used. The vacuum pump is used to lower the vacuum tank pressure. When the pump is turned on it consumes high power and can lower the overall system voltage, affecting boost control. Since it consumes power it also contributes to the overall fuel consumption and it is therefore of interest to include in a complete system model.

\subsection{Vacuum tank model}

The vacuum tank is modeled as an isothermal volume, with $T_{\text {tank }}=T_{a m b}$, and one state for the tank pressure. The pressure change in the tank is determined from the ideal gas law (11) and becomes

$\frac{d p_{\text {tank }}}{d t}=\frac{R T_{\text {amb }}}{V_{\text {tank }}}\left(\dot{m}_{\text {in }}-\dot{m}_{\text {out }}\right)$

The mass flow to the tank is the sum of the base leakage and the mass flow from each actuator. Mass flow out of the tank is achieved by the vacuum pump described in the next section. In Fig. 14, the measured and modeled tank pressures are shown for different operation of the three actuators. The left plots are from a series of steps in control signal and the right plots are from ramp responses. The pressure deviates slightly over time, but this is hard to avoid since the mass flow is integrated to get the tank pressure. A small error in flow will therefore result in an increasing pressure error. The leakage flow is overestimated in some operating points. This is not of major importance and the accuracy is enough to give a realistic behavior. If a more accurate estimate of the leakage flow during operation is required, an observer should be implemented. This model agreement is acceptable for a controls application, since there is a tank pressure sensor.

\subsection{Vacuum pump model}

The mass flow that the pump is able to produce is strongly connected to the pressure ratio over the pump, see Fig. 15. The data was collected by switching on the pump with close to ambient pressure in the tank and wait until the vacuum settled on the lowest possible pressure. The total mass flow was then calculated from the pressure trace and the base leakage flow to get the pump flow.

$\dot{m}_{\text {tank }}=\frac{a_{1}}{\frac{p_{\text {amb }}}{p_{\text {tank }}}+a_{2}}+a_{3}$

This three parameter model captures the mass flow as function of pressure ratio with good accuracy and its validation is included in Fig. 15.

\section{Model applications}

One important practical problem for control engineering is that the system voltage can vary several Volts during operation. This can directly influence the boost pressure controller performance. System voltage changes for example can be caused by switching on the vacuum pump. An example of the measured effect from a supply voltage disturbance (switching on light bulbs) is shown in Fig. 16. The change in the high pressure turbine actuator pressure, and the corresponding change in boost pressure before the throttle and high pressure stage turbo shaft speed is shown. The engine speed and throttle position were held constant during the experiment. During the first $2.5 \mathrm{~s}$ and after $20 \mathrm{~s}$ of the measurement, no light bulbs were lit. The difference in system supply voltage seen here is due to the high power vacuum pump being switched on initially in the measurement, resulting in different boost pressure.

\subsection{Actuator pressure model}

In Fig. 16, it can be seen that the system voltage variations of roughly $1.5 \mathrm{~V}$, lead to an actuator pressure change of $5 \mathrm{kPa}$. This pressure change causes the boost pressure to drop from $145 \mathrm{kPa}$ to $120 \mathrm{kPa}$, and turbo speed to drop from $125 \mathrm{krpm}$ to $90 \mathrm{krpm}$.

Also shown in the second plot of Fig. 16, is the simulated output of the high pressure stage turbine actuator model developed in the previous section, showing good agreement both during transients and stationary. The high pressure turbine 

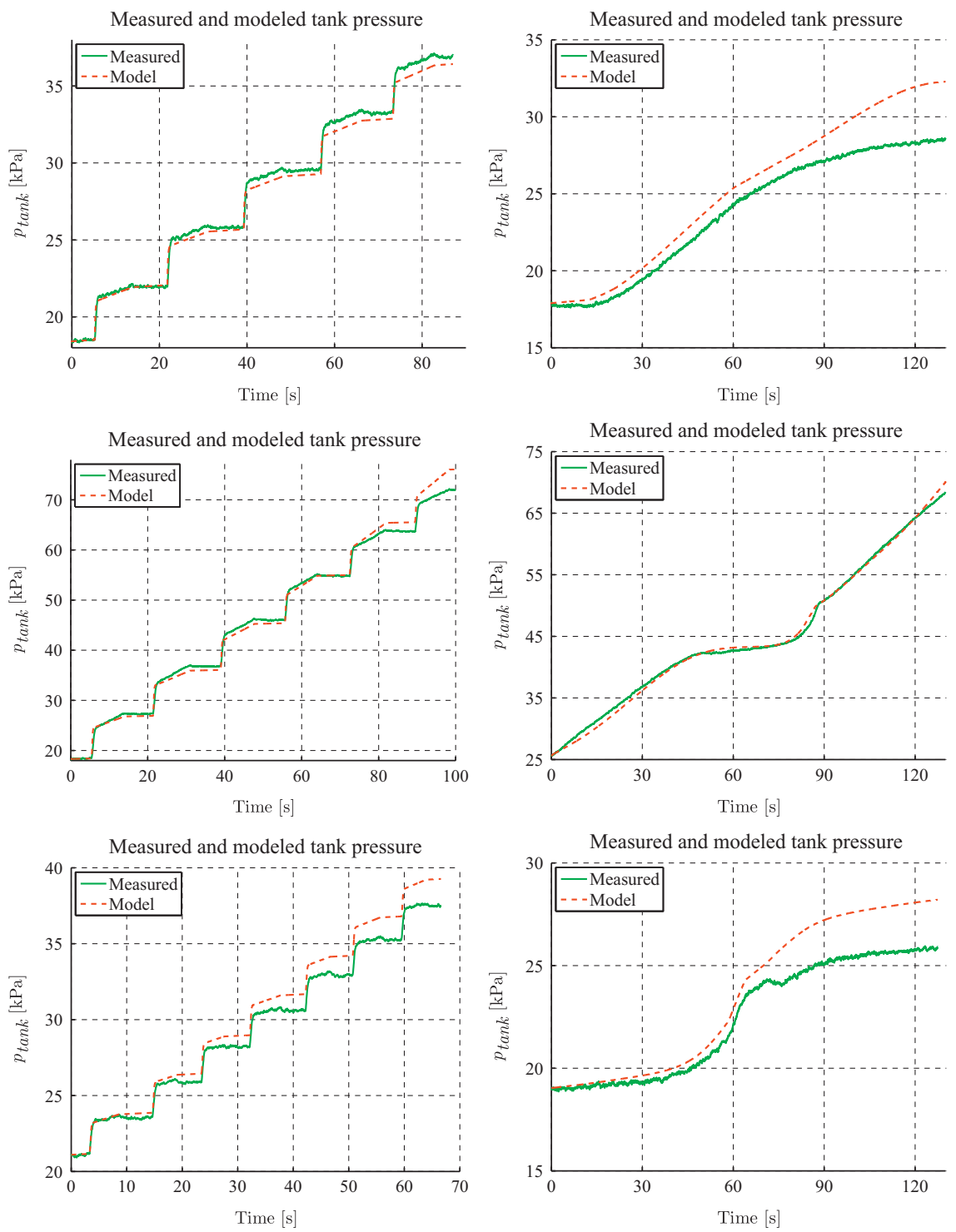

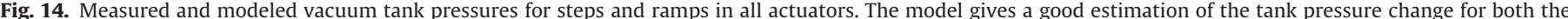

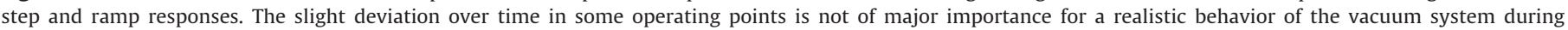
operation, and is hard to avoid since the system is integrating.

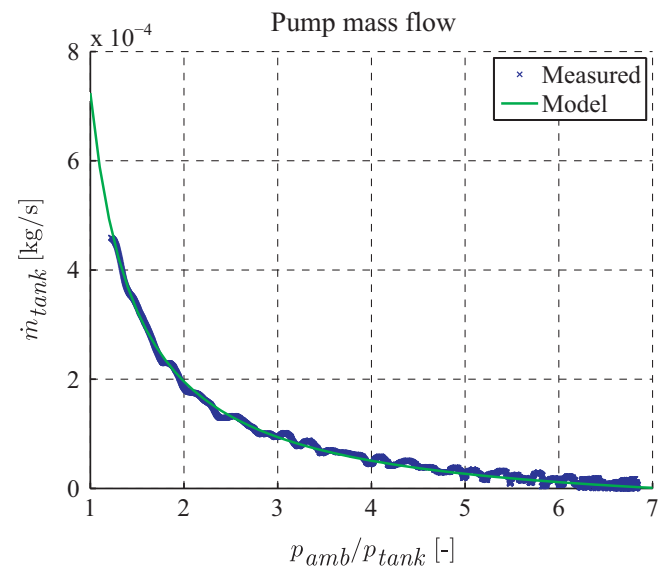

Fig. 15. Pump mass flow plotted against pressure ratio from ambient to vacuum tank. Also in the figure is a three parameter model of the pump flow as function of pressure ratio. actuator was chosen here, since this actuator was the most sensitive to supply voltage disturbances, due to steepness of the pressure to position curves, see Fig. 10. The behavior, however, is representable also for the low pressure stage turbine actuator as well as the high pressure stage compressor bypass actuator.

\subsection{Supply voltage compensation}

The system voltage affects the magnetic field controlling the plunger position in the solenoid valve. This is supported by the measurements and the developed control signal to pressure model in Section 3.1. It was shown that the dispersion due to system voltage could be removed by normalizing the control signal with the current system voltage. Based on the model and (1), the following modification to the boost controller output is proposed to handle deviations in supply voltage

$u_{c t r l, c o r r}=\frac{U_{\text {ref }}}{U_{\text {syst }}} u_{c t r l}$ 

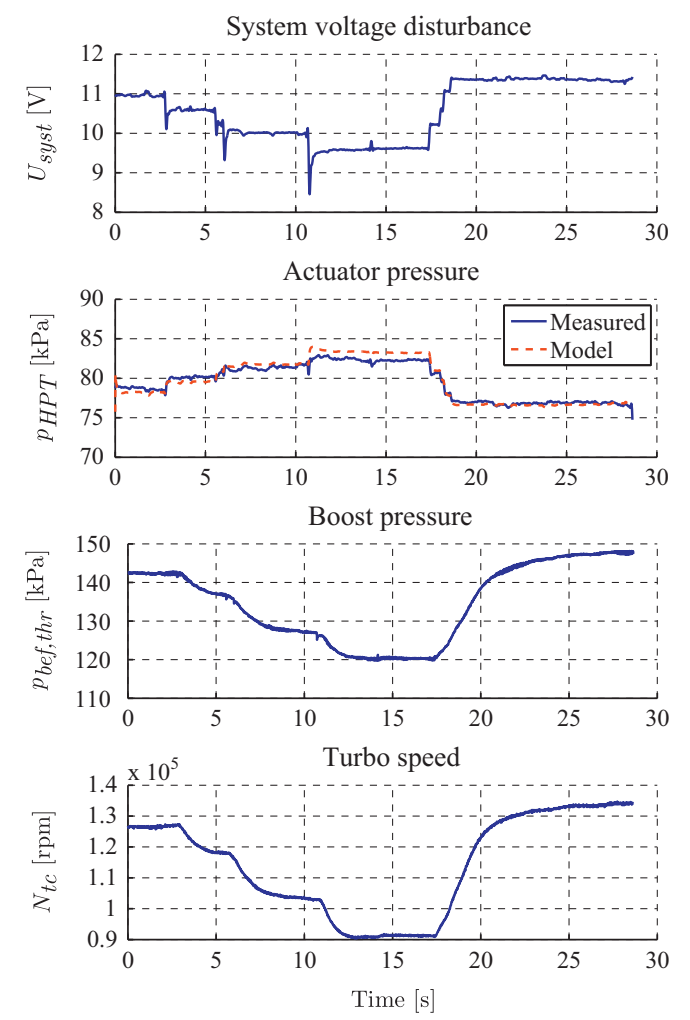

Fig. 16. The effect of a system voltage disturbance for a constant speed operating point, $1800 \mathrm{rpm}$, with fixed throttle position. Boost pressure is controlled by the smaller high pressure stage, and a series of voltage steps are applied. When the system voltage drops, the actuator pressure increases, the wastegate valve opens and the boost pressure decreases. The dashed line in the second plot shows that the developed model captures both the static and the dynamic behavior well.

where $u_{c t r l}$ is the controller output, $u_{c t r l, c o r r}$ is the corrected control signal, $U_{\text {syst }}$ is the supply voltage, and $U_{\text {ref }}$ is a reference voltage. Given a desired value of wastegate position, a corresponding control signal can be calculated using the inverse of the actuator model. A compensation for supply voltage is then calculated using (15). This was proposed and tested on the LPT actuator in Criscuolo et al. (2011) and has been extended and to include the HPT and HPC actuator as well. The compensation was tested with a voltage disturbance and the results are shown in Fig. 17, where open loop control with and without the voltage compensation has been subjected to the same disturbance. Despite the voltage disturbance, appropriately modifying the control signal with the aid of the developed model, the membrane position is kept almost constant, ensuring that the disturbance does not affect the boost pressure.

\subsection{Vacuum tank and pump model}

Another application of the developed system model is used to calculate the needed vacuum pressure, which in turn can be used to determine when it is necessary to run the vacuum pump. For example in the operating point in Fig. 16, a vacuum pressure of $70 \mathrm{kPa}$ would be enough for controlling the actuator. This can be related to the current conservative implementation that controls the pressure to always be below $35 \mathrm{kPa}$. Implementing a pump controller that tracks the needed pressure results in less usage of the pump and thereby energy savings.

It is also of interest to detect excess air leakage that could result from a broken pressure hose, increased valve or actuator leakages, or other malfunctions. Running under such conditions leads to excessive use of the vacuum pump, which increases fuel

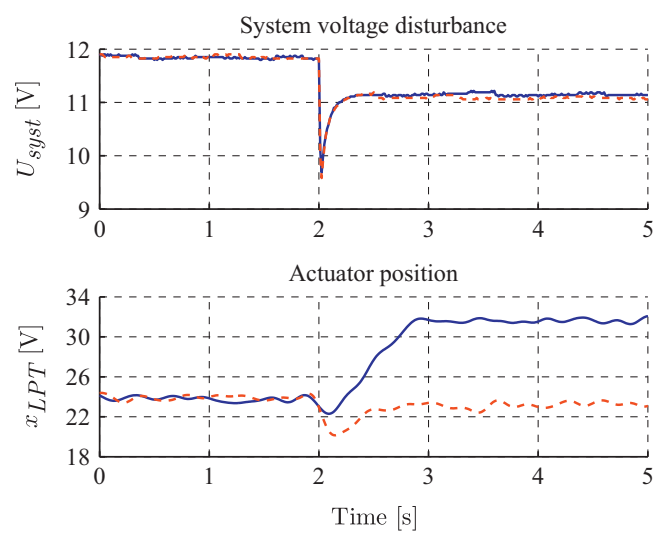

Fig. 17. Compensator performance for the supply voltage disturbance. The lower plot shows the wastegate position without compensator (solid) and with compensator (dashed). The compensated wastegate position is almost unaffected by the supply voltage disturbance.

consumption. Combining the tank pressure sensor and the developed model gives analytical redundancy and provides means for developing a residual and diagnosis system for detecting abnormal air leakages.

\subsection{Model based boost control}

As in the last example, the developed system model can be included in the feedforward loop of a model based boost control structure. An effective wastegate area reference (and thus position) can be calculated, based on required engine torque, surrounding conditions, and engine operating point, see for example Müller (2008), Hilding (2011), and Moulin and Chauvin (2011). Provided the position, the models developed here give the possibility to calculate the actuator control signal. A benefit of this feedforward is that disturbances due to surrounding conditions, like e.g. supply voltage, is effectively rejected. Combining this with for example the gain scheduled feedback in Criscuolo et al. (2011), enables integration of the system model in a complete model based boost control structure.

\section{Summary and conclusions}

A general modeling methodology has been developed and applied for a full vacuum actuation system used in turbocharged engines. The modeling covers the solenoid valves, pneumatic actuators, vacuum tank, and vacuum pump. The methodology is shown to work for all three actuators in a series sequential actuation system. Tank and pump models, with mass flow leakage, are shown to give a good description of the measurement data. The model parametrization methodology relies on low cost sensors and simple experiments. Significant variations are seen between the actuators, highlighting the need for a systematic method for determining parameters. It is observed that the solenoid valve can be described to function as a mechanical controller, which enabled the development of a compact yet accurate model for it. The model has a physical base which enables an engineer to develop model based control laws that account for changing conditions. The environment affects system performance, and the system voltage dependency is highlighted as important for control applications. The developed actuator model is the foundation for a nonlinear compensator, and it is demonstrated to be capable of rejecting system voltage disturbances. 


\section{Acknowledgments}

This research was supported by the VINNOVA Industry Excellence Center LINK-SIC.

\section{Appendix A. Nomenclature}

A description variables and subscripts used in the paper. Note that subscripts may be combined, for example $p_{L P T}$ refers to the pressure in the low pressure turbine actuator.

\begin{tabular}{|c|c|}
\hline Variable & Description \\
\hline$a$ & model parameter \\
\hline$b$ & friction coefficient \\
\hline$F$ & force \\
\hline$k$ & model parameter \\
\hline$m$ & mass \\
\hline$N$ & rotational speed \\
\hline$p$ & pressure \\
\hline$\Pi$ & pressure ratio \\
\hline$R$ & gas constant \\
\hline$T$ & temperature \\
\hline$U$ & voltage \\
\hline$u$ & control signal \\
\hline V & volume \\
\hline$x$ & position \\
\hline Subscript & Description \\
\hline act & actuator \\
\hline aero & aerodynamic \\
\hline$a m b$ & ambient \\
\hline C & compressor \\
\hline corr & corrected \\
\hline ctrl & control \\
\hline dyn & dynamic \\
\hline$f r$ & friction \\
\hline HP & high pressure \\
\hline$L P$ & low pressure \\
\hline $\mathrm{lim}$ & limit \\
\hline ref & reference \\
\hline$s p$ & spring \\
\hline stat & static \\
\hline$T$ & turbine \\
\hline tc & turbocharger \\
\hline vac & vacuum \\
\hline
\end{tabular}

\section{References}

Amirante, R., Innone, A., \& Catalano, L. (2008). Boosted PWM open loop control of hydraulic proportional valves. Energy Conversion and Management, 49, 2225-2236.

Andersson, P. (2005). Air charge estimation in turbocharged spark ignition engines. Ph.D. Thesis 989, Linköping University.

Borila, Y. (1988). A sequential turbocharging method for highly-rated truck diesel engines. SAE Technical Paper 860074.
Chasse, A., Moulin, P., Albrecht, A., Fontvielle, L., Guinois, A., \& Doléac, L. (2008). Double stage turbocharger control strategies development. SAE International Journal of Engines, 1, 636-646 2008-01-0988.

Criscuolo, I., Leufven, O., Thomasson, A., \& Eriksson, L. (2011). Model-based boost pressure control with system voltage disturbance rejection. In IFAC world congress. Milano, Italy.

Dahl, P. (1968). A solid friction model. Technical Report TOR-0158H3107-18I-1, The Aerospace Corporation.

Emmenthal, K., Hagermann, G., \& Hucho, W. (1979). Turbocharging small displacement spark ignited engines for improved fuel economy. SAE Technical Paper 790311.

Eriksson, L. (2007). Modeling and control of turbocharged SI and DI engines. Oil $\mathcal{E}$ Gas Science and Technology-Review of IFP, 62, 523-538.

Eriksson, L., Frei, S., Onder, C., \& Guzzella, L. (2002). Control and optimization of turbo charged spark ignited engines. In IFAC world congress.

Eriksson, L., \& Nielsen, L. (2000). Non-linear model-based throttle control. SAE Technical Paper 2000-01-0261.

Eriksson, L., Nielsen, L., Brugård, J., Bergström, J., Pettersson, F., \& Andersson, P. (2002). Modeling of a turbocharged SI engine. Annual Reviews of Control, 26, 129-137.

Galindo, J., Climent, H., Guardiola, C., \& Domenech, J. (2009a). Modeling the vacuum circuit of a pneumatic valve system. Journal of Dynamic Systems, Measurement, and Control, 131, 1-11.

Galindo, J., Climent, H., Guardiola, C., \& Domenech, J. (2009b). Strategies for improving the mode transition in a sequential parallel turbocharged automotive diesel engine. International Journal of Automotive Technology, 10 141-149.

Guzzella, L., Wenger, U., \& Martin, R. (2000). IC-engine downsizing and pressurewave supercharging for fuel economy. SAE Technical Paper 2000-01-1019.

Hendricks, E. (1989). The analysis of mean value engine models. SAE Technical Paper 890563.

Hilding, E. (2011). Enthalpy based boost pressure control. Master's Thesis, Linköping University, SE-581 83 Linköping.

Hlouvry, B., \& Dupont, P. (1994). A survey of models, analysis tools and compensation methods for the control of machines with friction. Automatica, 30, 1083-1138.

Mehmood, A., Laghrouche, S., \& El Bagdouri, M. (2010). Nonlinear modeling of the VNT pneumatic actuator with aero-dynamic force. In 6th IFAC symposium advances in automotive control.

Mehmood, A., Laghrouche, S., \& El Bagdouri, M. (2011). Modeling identification and simulation of pneumatic actuator for VGT system. Sensors and Actuators A: Physical, 165, 367-378.

Moulin, P., \& Chauvin, J. (2011). Modeling and control of the air system of a turbocharged gasoline engine. Control Engineering Practice, 19, 287-297.

Müller, M. (2008). Estimation and control of turbocharged engines. SAE Technical Paper 2008-01-1013.

Naseradinmousavi, P., \& Nataraj, C. (2011). Nonlinear mathematical modeling of butterfly valves driven by solenoid actuators. Applied Mathematical Modelling, 35, 2324-2335.

Nitta, J., Minato, A., \& Shimazaki, N. (2011). Performance evaluation of three-stage turbocharging system for heavy-duty diesel engine. SAE Technical Paper 201101-0374.

Olsson, H., Åström, K. J., Canudas de Wit, C., Gáfvert, M., \& Lischinsky, P. (1998) Friction models and friction compensation. European Journal of Control, 4 176-195.

Petitjean, D., Bernardini, L., Middlemass, C., Shahed, S., \& Hurley, R. (2004). Advanced gasoline engine turbocharging technology for fuel economy improvements. SAE Technical Paper 2004-01-0988.

Singh, R., \& Kunt, C. (1990). A linear time varying model for on-off valve controlled pneumatic actuators. Transactions of the ASME.

Taghizadeh, M., Ghaffari, A., \& Najafi, F. (2009). Modeling and identification of a solenoid valve for PWM control applications. Comptes Rendus Mecanique, 337, 131-140.

Topcu, E. E., Yüksel, I., \& Kamis, Z. (2006). Development of electro-pneumatic fast switching valve and investigation of its characteristics. Mechatronics, 16 365-378.

Wang, J., Pu, J., \& Moore, P. (1999). A practical control strategy for servo-pneumatic actuator systems. Control Engineering Practice, 12, 1483-1488.

Wang, X.-S., Cheng, Y.-H., \& Peng, G.-Z. (2007). Modeling and self-tuning pressure regulator design for pneumatic-pressure-load systems. Control Engineering Practice, 15, 1161-1168.

Zhang, Z., Deng, K., Wang, Z., \& Zhu, X. (2008). Experimental study on the threephase sequential turbocharging system with two unequal size turbochargers. SAE International Journal of Fuels and Lubricants, 1, 1181-1186 2008-01-1698. 